\title{
A theoretical study of the sequence of failure in precast panel shear walls
}

\author{
B. STAFFORD SMITH \& K. M. K. RAHMAN
}

Dr K. Alhashimi, Department of Civil Engineering, University of Aston in Birmingham

The Authors' work is in many ways similar to the work carried out at the universities of Birmingham and Aston in Birmingham on crack propagation through stabilized soil and infill panels. ${ }^{2}$ Like all work on non-linear materials, such as concrete, the Authors' calculation on separation of crack propagation is simplified to the extent that it is doubtful whether it will agree well with practical cases.

43. I would like to suggest that the word 'failure' should not be used in place of the term 'physical separation of the panels', as failure means the loss of stiffness of the entire structure. Mathematically failure occurs when the determinant of the overall stiffness matrix of the entire structure reduces to zero, which is not the case when two elements separate. The Authors do not mention this fact. Do they carry out a stability test of this kind each time they introduce a separation into the structure? If not, what criterion do they use to detect the final state of collapse?

44. In their calculation of the state of failure due to shear, the Authors introduce the constant $K$ which depends on the location of the point where failure occurs, the geometry of the wall and so on. However, they do not indicate how $K$ is estimated, nor do they point out the empirical nature of this constant.

45. In the finite element analysis of the walls, the Authors assume that separation does not occur within a panel. This assumption is not necessary and the method can be extended to cater for cases where fracture of a panel may take place. This assumption was perhaps adopted so that large, panel-size elements could be used in the analysis. This indeed reduces the accuracy of the analysis.

46. When separation takes place between two elements, the Authors renumber the nodes. This is unnecessary. At the University of Aston in Birmingham a double or multiple joint numbering system has been adopted. For instance, the model shown in Fig. 7(a) is numbered so that a joint, where a crack can take place, is defined by two node numbers. In this model nodes 5 and 9 are initially the active nodes to which elements 1, 2, 3 and 4 are connected. However, nodes 6 and 10 are dummy nodes with no degrees of freedom. Before separation takes place the overall stiffness matrix has no rows or columns for these dummy nodes and they in no way influence the analysis. When a crack takes place between nodes 5 and 6 , for instance (Fig. 7(b)), node 6 is rendered active. Elements 1 and 3 are then connected to node 5 and elements 2 and 4 are connected to node 6 .

47. When considering tensile failure of the vertical joint, the Authors apply equal and opposite holding forces representing the strength of the yielding steel. A more elegant approach would be one similar to that previously adopted ${ }^{3-5}$ in the elasticplastic analysis of frames. The stress-strain relationship of the yielding steel is simply taken to be elastic-plastic. Then, wherever the steel yields, its yield force is included in the overall stiffness equations of the structure.

48. By taking panel-size rectangular elements, the Authors not only restrict themselves to large elements, but also are forced to introduce a sudden separation along the entire length of a panel. This leads to an underestimation of the failure load. At Aston a higher order-isoparametric elements-has been adopted (Fig. 8). The advantages are as follows

Paper published: Proc. Instn Civ. Engrs, Part 2, 1973, 55, Sept., 581-592. 


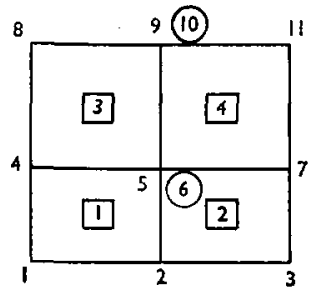

(a)

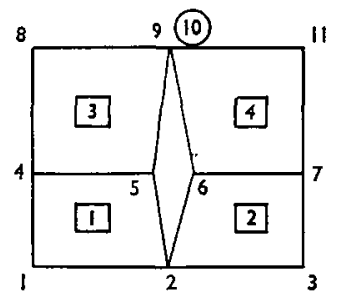

(b)

Fig. 7. Number of joints in a model (a) before separation, (b) after separation

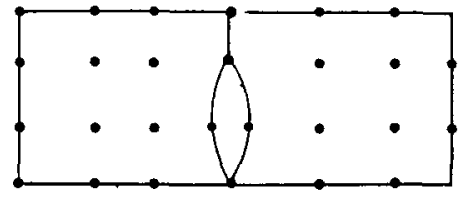

(a)

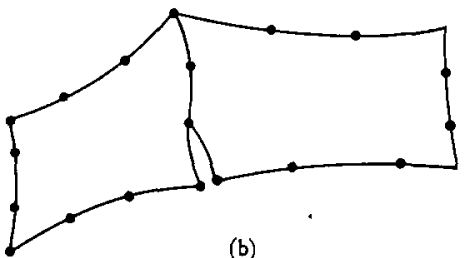

(b)

Fig. 8. Isoparametric elements; (a) rectangular isoparametric elements with a partial intermediate separation of elements, (b) irregular isoparametric elements with edges drawn along the expected separation lines

(a) The elements can have irregular shape and element boundaries can be positioned more realistically to coincide with the expected crack locations.

(b) Larger elements can be used without loss of accuracy.

(c) More significant, these elements have intermediate nodes along their edges.

49. Thus it is not necessary to introduce cracks along the entire length of an element. It is understood that the process of separation between elements takes place gradually and separation extends only to places where the separation stress is equal to the separation strength of the material. Thus with isoparametric elements, cracks are allowed to develop at any node and extend only along a part of the edge of an element to the adjacent nodes along the element side. This improves the accuracy of the analysis. These intermediate nodes have the added advantage of representing the applied loads more realistically. The Authors' comments on these points would be appreciated.

\section{Dr Stafford Smith and Dr Rahman}

Dr Alhashimi's criticisms relate mainly to details of the method of analysis and their effect on the accuracy of the solutions. The primary purposes of the investigation were to explore the possibility of a reserve of strength in the shear wall beyond the load at initial failure and to obtain a rough estimate of the magnitude of the reserve strength. Although greater accuracy in an analysis is always desirable, the relatively crude starting assumptions would probably make any improved accuracy from a more sophisticated approach only notional and perhaps misleading. Further, the additional computer effort of using more sophisticated elements in such an iterative finite element analysis would have been prohibitively expensive. For these reasons the simpler approach was used. 
51. A more appropriate definition of failure than that suggested by Dr Alhashimi is that stage at which a structure or part of a structure behaves unacceptably. In this case when a joint separates or slides it is considered to have failed, and is described as such in the Paper. At the beginning of each new cycle a check on the stability of the whole system of panels, similar to that mentioned by Dr Alhashimi, was carried out. In each cycle a check on the compressive stresses at each joint was also made. Inadequacy on either of these points would have defined failure of the whole structure; however, in the practical examples considered the ultimate load of the structure was reached many stages before the equilibrium of the system deteriorated.

52. The value of $K$ at each node in each cycle was determined from the results of the unit wind load analysis by substitution in equation (3). The value of $K$ is not empirical but has a definite value for a particular joint in the modified structure at a certain stage of disintegration.

53. The assumption of failure within joints only is a realistic assumption which allows a simplified approach to the problem. To include the possibility of failure within the panels would not only add substantially to the complexity of the analysis but would also raise the problem of suitable failure criteria for the panel material, apart from ignoring experimental evidence pointing to the high probability of failure in the joints.

54. Dr Alhashimi suggests that elastic-plastic consideration of the reinforcement would have been more elegant. The stiffness of the reinforcement was not included in the analysis because the reinforcement is almost entirely contained within the panels. Only when the force between the panels exceeded the yield strength of the steel was the reinforcement brought into consideration. At that stage it was appropriately represented by forces equal to its yield strength.

55. We appreciate that more elements or elements with mid-side nodes would have given a more refined consideration of the separation between elements. It would be interesting to see the effect of such a refinement on the pattern of cracking. However, in the vertical joint a major part of the reinforcement is concentrated at each floor level; therefore, for tensile failure of the vertical joint, it was probably more realistic to use the coarse separation adopted.

\section{References}

2. Alhashimi K. Deformation and fracture in cement stabilized soil. PhD thesis, University of Birmingham, 1972.

3. MaJiD K. 1. Non-linear structures. Butterworth, 1972.

4. MAJID K. I. and ANDERSON D. The computer analysis of large multistorey framed structures. Struct. Engr, 1968, 46, Nov., 357-365.

5. MAJID K. I. and ONEN Y. H. The elasto-plastic failure load analysis of complete building structures. Proc. Instn Civ. Engrs, Part 2, 1973, 55, Sept., 619-634. 